\title{
Large deviation function for the entropy production: Optimal trajectory and role of fluctuations
}

\author{
Thomas Speck \\ Institut für Theoretische Physik II, Heinrich-Heine-Universität Düsseldorf, \\ Universitätsstraße 1, 40225 Düsseldorf, Germany
}

\author{
Andreas Engel \\ Universität Oldenburg, Institut für Physik, 26111 Oldenburg, Germany \\ Udo Seifert \\ II. Institut für Theoretische Physik, Universität Stuttgart, Pfaffenwaldring 57, 70550 \\ Stuttgart, Germany
}

\begin{abstract}
We study the large deviation function for the entropy production rate in two driven one-dimensional systems: the asymmetric random walk on a discrete lattice and Brownian motion in a continuous periodic potential. We compare two approaches: the Donsker-Varadhan theory and the Freidlin-Wentzell theory. We show that the wings of the large deviation function are dominated by a single optimal trajectory: either in forward (positive rate) or in backward direction (negative rate). The joining of both branches at zero entropy production implies a non-differentiability and thus the appearance of a "kink". However, around zero entropy production many trajectories contribute and thus the kink is smeared out.
\end{abstract}




\section{Contents}

1 Introduction $\quad 2$

2 Asymmetric random walk 4

2.1 Donsker-Varadhan theory . . . . . . . . . . . . . . . . 4

2.2 Alternative derivation . . . . . . . . . . . . . . . . 6

3 Driven Brownian motion $\quad 7$

3.1 Donsker-Varadhan theory . . . . . . . . . . . . . 8

3.2 Stochastic action and effective potential . . . . . . . . . . . 8

3.3 Optimal trajectory . . . . . . . . . . . . . . . . 10

3.4 Small-noise limit . . . . . . . . . . . . . . . . . . . . . . . . . 12

3.5 Role of fluctuations . . . . . . . . . . . . . . . . . . . 13

3.5.1 Gaussian fluctuations. . . . . . . . . . . . . . . . 14

3.5.2 Dilute instanton gas. . . . . . . . . . . . . . . . 14

4 Conclusions $\quad 16$

AppłominetAtheory $\quad 16$

\section{Introduction}

Driving a system away from thermal equilibrium implies a non-vanishing entropy production rate in the surrounding environment due to heat dissipation. For mesoscopic systems, the energy thus exchanged with the environment is a stochastic quantity, and so is the entropy $s_{\mathrm{m}}$ produced along a single trajectory. This observation forms the basis of stochastic thermodynamics [1]. For a system driven into a non-equilibrium steady state, the long-time limit of the corresponding probability distribution $p\left(s_{\mathrm{m}}, t\right)$ defines a large deviation function $(\mathrm{LDF})$

$$
h(\sigma) \equiv \lim _{t \rightarrow \infty}-\frac{\ln p\left(s_{\mathrm{m}}, t\right)}{\left\langle\dot{s}_{\mathrm{m}}\right\rangle t}
$$

with normalized entropy production rate

$$
\sigma \equiv \frac{s_{\mathrm{m}}}{\left\langle\dot{s}_{\mathrm{m}}\right\rangle t}
$$

Under very general assumptions, this large deviation function obeys the GallavottiCohen symmetry [2, 3, 4]

$$
h(-\sigma)=h(\sigma)+\sigma .
$$

Experimental tests of this relation have been performed in a variety of systems, e.g., a single trapped colloidal particle [5, 6] and a mechanical oscillator [7]. Other large deviation functions also play an important role for the study of non-equilibrium systems [8, 9, 10, 11]. 

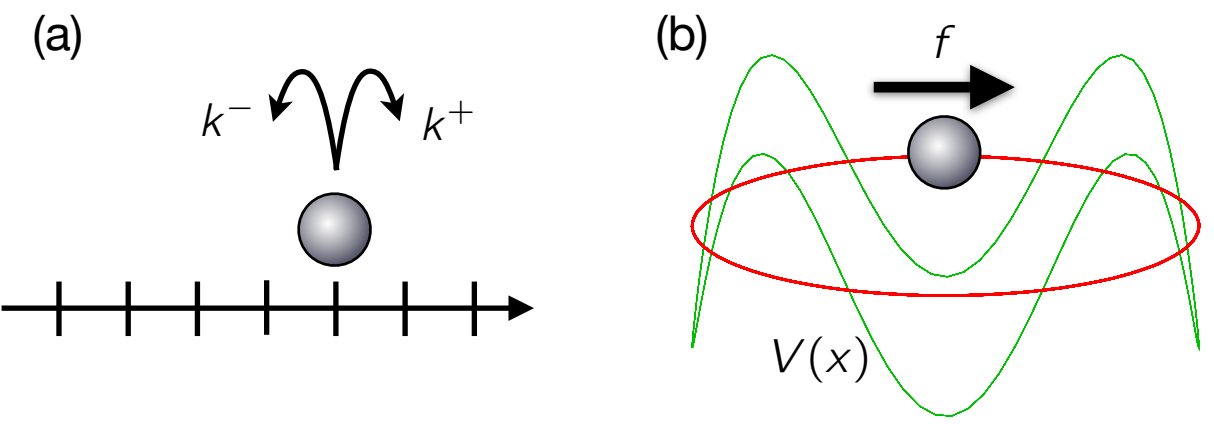

Figure 1. The two systems studied: (a) the asymmetric random walk of a particle on a lattice with discrete positions. Different forward rate $k^{+}$and backward rate $k^{-}$can be interpreted as an effective driving force $f=\ln \left(k^{+} / k^{-}\right)$. (b) The continuous analog is a particle moving in a periodic ring potential $V(x)$ and driven by a force $f$.

We study two paradigmatic models for driven systems sketched in Fig. 1; the asymmetric random walk on a discrete lattice and Brownian motion of a colloidal particle in a one-dimensional periodic potential. The latter system has been studied extensively in the context of the fluctuation-dissipation theorem [12, 13, 14, 15, 16]. In order to obtain explicit expressions for Eq. (1), two main approaches are discussed in the literature (for a comprehensive review and references, see Ref. [17]). The first is the Donsker-Varadhan theory, stating that the scaled cumulant generating function

$$
\alpha(\lambda) \equiv \lim _{t \rightarrow \infty} \frac{1}{t} \ln \left\langle e^{\lambda s_{\mathrm{m}}}\right\rangle
$$

is related to the LDF through the Fenchel-Legendre transformation

$$
h(\sigma)=\sup _{\lambda}\left\{\lambda \sigma-\alpha(\lambda) /\left\langle\dot{s}_{\mathrm{m}}\right\rangle\right\} .
$$

This method has been used previously to obtain the LDF for the two systems we study here: the asymmetric random walk [4] and the continuous periodic potential [18]. The second approach due to Freidlin and Wentzell [19] focusses on paths instead of the scaled cumulant generating function $\alpha(\lambda)$. The probability of a path $x(\tau)$ obeys a large deviation principle

$$
P[x(\tau)] \sim e^{-J[x(\tau)] / \varepsilon}
$$

in the limit of small noise $\varepsilon \rightarrow 0$. The large deviation function $h(\sigma)$ can then be obtained from the optimal trajectory $x_{*}(\tau)$ minimizing $J[x(\tau)]$ using the contraction principle. While the Donsker-Varadhan theory makes no assumptions on the noise strength and is thus more general, it is also less intuitive. The Freidlin-Wentzell theory, on the other hand, is more appealing to physical intuition since it allows a more direct insight.

The purpose of this work is two-fold: First, we provide a detailed study comparing for two specific models the two approaches to the calculation of the large deviation function. Based on the Freidlin-Wentzell approach, the second purpose is to study the origin of a particular feature of the LDF: The emergence of a "kink" around zero entropy production, an abrupt albeit differentiable change of the slope [18]. This 
"kink" has been observed in distributions of the velocity (which is closely related to the entropy production) in models for molecular motors [20], and experimentally for a tagged granular particle [21]. Of course, this "kink" does not indicate a phase transition. Dynamic phase transitions are indicated by a jump discontinuity of either the first or second derivative of the scaled cumulant generating function $\alpha(\lambda)$. Such transitions have been found in interacting systems, e.g., for the totally asymmetric exclusion process [22], the symmetric exclusion process [23], and for models with kinetic constraints [24].

Basically two theoretical explanations for the physical origin of the kink have been proposed so far. Pleimling and coworkers [25, 26] have claimed that the kink is a generic feature of LDFs for the entropy production that follows quite generally from the Gallavotti-Cohen symmetry Eq. (3). But the relation Eq. (3) only restricts the anti-symmetric part, i.e., it allows us to write $h(\sigma)=h_{\mathrm{s}}(\sigma)-\sigma / 2$ with $h_{\mathrm{s}}(-\sigma)=h_{\mathrm{s}}(\sigma)$. Clearly, from Eq. (3) no statement can be inferred about $h_{\mathrm{s}}$, which is a non-universal function containing the details of the dynamics and the driving. On the other hand, the anti-symmetric part is universal and originates from breaking time-reversal symmetry. As an alternative explanation, Budini [27] has attributed the kink to "intermittency", i.e., the random switching between different dynamic regimes. Here we show explicitly that the optimal trajectory minimizing the stochastic action indeed corresponds to two different regimes depending on the sign of $\sigma$ : forward $(\sigma>0)$ or backward $(\sigma<0)$. Considering only the optimal trajectories, therefore, implies a non-differentiability in the large deviation function at $\sigma=0$, which, however, is smeared out by fluctuations.

\section{Asymmetric random walk}

We first consider a particle on an infinite one-dimensional lattice. The particle can hop to the left with rate $k^{-}$, or hop to the right with rate $k^{+}$. A single trajectory of length $t$ is characterized by the number of hops to the left, $n^{-}$, and to the right, $n^{+}$. Travelling the distance $n \equiv n^{+}-n^{-}$, the entropy produced in the surrounding medium is

$$
s_{\mathrm{m}}\left(n^{+}, n^{-}\right)=n \ln \frac{k^{+}}{k^{-}} \equiv n f
$$

where

$$
\left\langle\dot{s}_{\mathrm{m}}\right\rangle=f\left(k^{+}-k^{-}\right)=f k^{+}\left(1-e^{-f}\right)
$$

is the mean entropy production rate. For $k^{+}=k^{-}$the rates obey detailed balance and clearly no entropy is produced on average.

\subsection{Donsker-Varadhan theory}

In a seminal paper on the fluctuation theorem for stochastic dynamics 4], Lebowitz and Spohn have obtained the LDF for the asymmetric random walk (ARW) from the cumulant generating function. The normalized function $h(\sigma)$ can be cast in the form

$$
h(\sigma)=(f z)^{-1}\left\{\cosh (f / 2)+z \sigma[\operatorname{arsinh}(z \sigma)-f / 2]-\sqrt{(z \sigma)^{2}+1}\right\}
$$



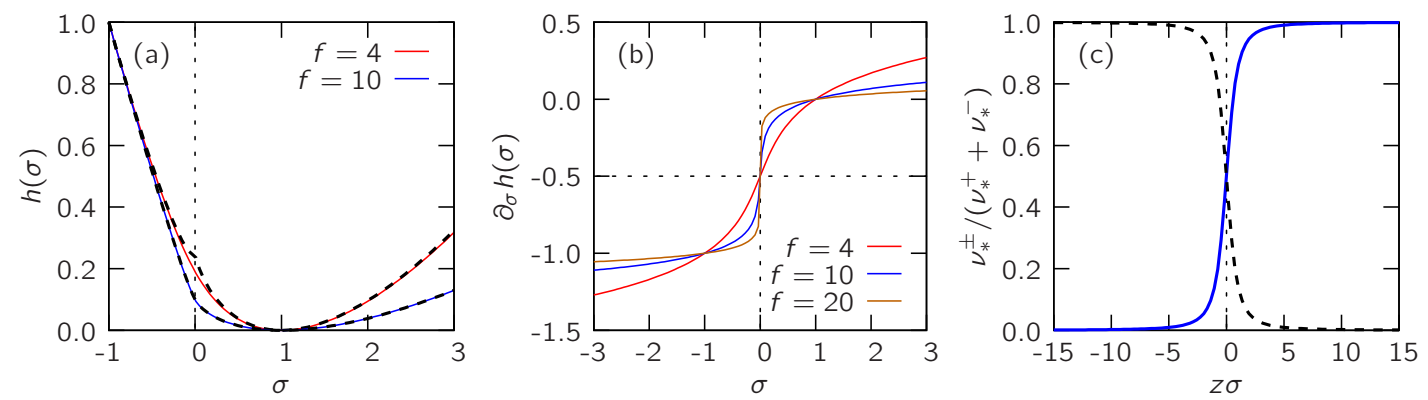

Figure 2. Asymmetric random walk: (a) Large deviation function $h(\sigma)$ for two forces $f=4$ and $f=10$ (solid lines). Also shown is the limiting form Eq. 12 (dashed lines). (b) The derivative becomes steeper at $\sigma=0$ for higher driving force $f$. (c) The forward (solid line) and backward (dashed line) fluxes [Eq. [18]] vs. the scaled rate $z \sigma$, where $z=\sinh (f / 2)$.

with $z \equiv \sinh (f / 2)$. It is straightforward to check that the function $h(\sigma)$ has the following properties: (i) it is convex and non-negative, $h(\sigma) \geqslant 0$, with minimum $h(1)=0$, (ii) it obeys the Gallavotti-Cohen symmetry Eq. (3), and (iii), following from this symmetry, $h(-1)=1$ holds.

To make our introductory comments concrete about a kink in the large deviation function, for $|z \sigma| \gg 1$ we expand the inverse hyperbolic sine leading to

$$
h(\sigma) \approx(f z)^{-1}\{\cosh (f / 2)+z \sigma[ \pm \ln z|\sigma|-f / 2]-z|\sigma|\} .
$$

The plus sign holds for $\sigma>0$ while the minus sign holds for $\sigma<0$. Here and in the following we assume $f>0$. Considering the case $f \gg 1$ of large asymmetry we can further simplify

$$
\frac{\cosh (f / 2)}{z} \approx 1, \quad \ln z \approx-\frac{f}{2}
$$

yielding the limiting form

$$
h(\sigma) \approx \frac{1}{f} \begin{cases}1+\sigma(\ln \sigma-1) & (\sigma>0) \\ 1+|\sigma|(\ln |\sigma|-1+f) & (\sigma<0)\end{cases}
$$

of the large deviation function in agreement with Ref. [25]. In particular, the first derivative

$$
h^{\prime}(\sigma) \approx \frac{1}{f} \begin{cases}\ln \sigma & (\sigma>0) \\ -\ln |\sigma|-f & (\sigma<0)\end{cases}
$$

jumps at $\sigma=0$. However, this jump is only apparent since for the expansion leading to the limiting expression Eq. (12) we used $|\sigma| \gg 1 / z$, i.e., we exclude a region of size $1 / z$ around $\sigma=0$. With increasing $f$ this region becomes smaller but it does not vanish. Hence, for any finite $f$ the function $h(\sigma)$ is differentiable. In Fig. 2, the large deviation function and its first derivative are plotted for different forces $f$. 


\subsection{Alternative derivation}

We now give an alternative derivation of Eq. (9) in the spirit of Freidlin-Wentzell. The path weight of a single trajectory is

$$
P\left(n^{+}, n^{-} ; t\right)=\left(\begin{array}{c}
m \\
n^{+}
\end{array}\right) \frac{t^{m}}{m !}\left(k^{+}\right)^{n^{+}}\left(k^{-}\right)^{n^{-}} e^{-\left(k^{+}+k^{-}\right) t}
$$

with $m \equiv n^{+}+n^{-}$total jumps. This weight fulfills the normalization condition

$$
\sum_{m=0}^{\infty} \sum_{n^{+}=0}^{m} P\left(n^{+}, n^{-} ; t\right)=1 \text {. }
$$

Introducing dimensionless fluxes $\nu^{ \pm} \equiv n^{ \pm} /\left(k^{+} t\right)$ and expanding the factorials using Stirling's approximation we obtain the rate function

$$
\begin{aligned}
J\left(\nu^{+}, \nu^{-}\right) & \equiv-\frac{1}{t} \ln P \\
& =k^{+}\left[1+e^{-f}-\left(\nu^{+}+\nu^{-}\right)+f \nu^{-}+\nu^{+} \ln \nu^{+}+\nu^{-} \ln \nu^{-}\right],
\end{aligned}
$$

which does not depend on time explicitly. For long times $t$ the path weight Eq. (14) is sharply peaked around the minimum of $J$. We will call trajectories that contribute to this minimum "optimal" trajectories. Note that the small parameter $\varepsilon$ in Eq. (6) is now given by $\varepsilon=1 / t$, i.e., here the time limits the fluctuations around the minimum.

For fixed $\sigma$, forward and backward fluxes are not independent but related through

$$
\nu^{+}=\nu^{-}+\left(1-e^{-f}\right) \sigma .
$$

From $\partial J / \partial \nu^{-}=0$ we then find that the minimum of $J$ is reached for fluxes

$$
\nu_{*}^{ \pm}=e^{-f / 2}\left[\sqrt{(z \sigma)^{2}+1} \pm z \sigma\right], \quad z \equiv \sinh (f / 2) .
$$

Using that

$$
\ln \nu_{*}^{ \pm}=-\frac{f}{2} \pm \operatorname{arsinh}(z \sigma)
$$

it is straightforward to show that $J\left(\nu_{*}^{+}, \nu_{*}^{-}\right) /\left\langle\dot{s}_{\mathrm{m}}\right\rangle=h(\sigma)$ yields the result Eq. (9) obtained from the Donsker-Varadhan theory.

The advantage of the alternative derivation is that it becomes transparent which trajectories contribute to $h(\sigma)$ for a given normalized entropy production rate $\sigma$. We now use this insight to discuss the physical origin of the apparent kink. What does the optimal trajectory to reach a specified entropy production, i.e., the trajectory that minimizes the rate function $J$, look like? As becomes clear from Eq. (18), for a given positive $\sigma \gg 1 / z$ the optimal trajectory consists almost entirely of forward jumps. Conversely, for negative $\sigma$ the optimal trajectory consists almost entirely of backward jumps even though these trajectories are extremely unlikely. This might seem surprising but any forward jump has to be compensated by an unlikely backward jump. Setting $n^{-}=0$ for $\sigma>0$ and $n^{+}=0$ for $\sigma<0$ leads to the two branches of the limiting form given in Eq. (12). The reason why nevertheless $h(\sigma)$ is differentiable is that for $|\sigma| \lesssim 1 / z$ many trajectories contribute to the large deviation function. In particular, 
for $\sigma=0$ the forward and backward fluxes become equal, $\nu^{+}=\nu^{-}$. All trajectories with the same number of forward and backward jumps contribute since they have the same weight. Their number is given by the binomial coefficient in Eq. (14). In contrast, there is only one optimal combination $\left(n^{+}, n^{-}\right)$in the wings of the distribution.

\section{Driven Brownian motion}

In this section we consider a single colloidal particle moving in a closed ring geometry with circumference $L$ and external periodic potential $V(x+L)=V(x)$. The overdamped dynamics are described by the Langevin equation

$$
\dot{x}=\mu_{0}\left[-V^{\prime}(x)+f\right]+\eta,
$$

where the prime denotes the derivative with respect to $x$. The particle is driven out of equilibrium through the force $f$ resulting in a non-vanishing current through the ring. The noise $\eta$ describes the effective interactions between the solvated particle and the solvent at temperature $T$ with correlations

$$
\left\langle\eta(\tau) \eta\left(\tau^{\prime}\right)\right\rangle=2 D_{0} \delta\left(\tau-\tau^{\prime}\right)
$$

Through $D_{0}=\mu_{0} T$ we assume that the solvent remains at equilibrium even though the particle is driven. To simplify the notation we will use dimensionless quantities

$$
x \mapsto L x, \quad V(x) \mapsto T V(x), \quad f \mapsto \frac{T}{L} f, \quad \tau \mapsto \frac{L^{2}}{D_{0}} \tau
$$

throughout the remainder of this paper. Moreover, these units make transparent the connection with the ARW. In particular, the number of periods traversed is $n$ and the dimensionless force $f$ corresponds to the force defined in Eq. (7). We will also need the notion of a critical force $f_{\mathrm{c}}$ such that for $f>f_{\mathrm{c}}$ the deterministic force $-V^{\prime}(x)+f>0$ is positive for all $x$ and, therefore, in the absence of noise running solutions exist.

The rate of entropy production is $\dot{s}_{\mathrm{m}}=f \dot{x}$ with $s_{\mathrm{m}}=f \Delta x$ [cf. Eq. (7) for the ARW], where $\Delta x$ is the distance the particle has traveled during the time $t$. The mean velocity of the particle can be calculated from Stratonovich's formula [28]

$$
\langle\dot{x}\rangle=\kappa\left(1-e^{-f}\right)
$$

with

$$
\kappa^{-1} \equiv \int_{0}^{1} \mathrm{~d} x \int_{0}^{1} \mathrm{~d} z \exp \{V(x)-V(x-z)-f z\} .
$$

The mean velocity (and also the mean entropy production) of ARW and ring potential agree if we set $k^{+}=\kappa$, cf. Eq. (8). However, in general fluctuations will be different, leading to different shapes for the LDFs. For large forces $f$ the influence of the potential on the particle motion becomes negligible and $\langle\dot{x}\rangle \approx f$. 


\subsection{Donsker-Varadhan theory}

The time evolution of the probability distribution of $x$ is governed through the FokkerPlanck operator

$$
\hat{L}_{0} \equiv \frac{\partial}{\partial x}\left[\left(V^{\prime}-f\right)+\frac{\partial}{\partial x}\right] .
$$

In particular, the steady state distribution obeys $\hat{L}_{0} p_{\mathrm{ss}}(x)=0$. To calculate $\alpha(\lambda)$ [Eq. (4)] consider the two-sided Laplace transform

$$
\tilde{p}(x, \lambda, t)=\int_{-\infty}^{+\infty} \mathrm{d} s_{\mathrm{m}} e^{\lambda s_{\mathrm{m}}} p\left(x, s_{\mathrm{m}}, t\right)
$$

of the joint probability $p\left(x, s_{\mathrm{m}}, t\right)$ to find the particle at position $x$ with an amount of entropy $s_{\mathrm{m}}$ produced up to time $t$. The time evolution of the transformed joint probability obeys $\partial_{t} \tilde{p}=\hat{L}_{\lambda} \tilde{p}$, where the operator

$$
\hat{L}_{\lambda}=\hat{L}_{0}+\left[2 \frac{\partial}{\partial x}+V^{\prime}-f\right](f \lambda)+(f \lambda)^{2}
$$

has been determined in Ref. [18]. We expand the joint probability in eigenfunctions of the time evolution operator

$$
\tilde{p}(x, \lambda, t)=\sum_{i=0}^{\infty} c_{i} e^{\alpha_{i}(\lambda) t} \psi_{i}(x, \lambda),
$$

where the coefficients $c_{i}$ quantify the overlap with the initial distribution $\tilde{p}(x, \lambda, 0)=$ $p_{\text {ss }}(x)$. The eigenvalue equations read $\hat{L}_{\lambda} \psi_{i}(x, \lambda)=\alpha_{i}(\lambda) \psi_{i}(x, \lambda)$. In the limit of long times, the joint probability is dominated by the largest eigenvalue $\alpha_{0}$. Integrating Eq. 26) over the position $x$ thus leads to

$$
\left\langle e^{\lambda s_{\mathrm{m}}}\right\rangle \sim e^{\alpha_{0}(\lambda) t},
$$

and we conclude that the scaled cumulant generating function $\alpha(\lambda)=\alpha_{0}(\lambda)$ is indeed given by the largest eigenvalue of the time evolution operator (27). It can be calculated solving either the eigenvalue problem directly [18] or through the Ritz variational method [29]. The function $h(\sigma)$ then follows from (5). Here we use the first method and refer to Ref. [18] for further details.

\subsection{Stochastic action and effective potential}

To continue we employ the well-established machinery of path integrals for stochastic

processes [30] that has been developed to deal, e.g., with diffusion in bistable potentials [31] and escape problems [32]. Imagine that the particle starts at position $x_{0}$ and travels a distance $\Delta x$ during the time $t$. The probability for such a transition

$$
P\left(x_{0}+\Delta x \mid x_{0} ; t\right)=\int_{x_{0}}^{x_{0}+\Delta x}[\mathrm{~d} x(\tau)] e^{-\mathcal{S}[x(\tau)]}
$$

is obtained through summing over all possible paths with stochastic action

$$
\mathcal{S}[x(\tau)]=\int_{0}^{t} \mathrm{~d} \tau\left\{\frac{1}{4}\left(\dot{x}+V^{\prime}-f\right)^{2}-\frac{1}{2} V^{\prime \prime}\right\}
$$


calculated along a single path $x(\tau)$. The last term $V^{\prime \prime} / 2$ is the Jacobian arising from the change of variables $\eta \rightarrow x$.

The stochastic action Eq. (31) can be rearranged to give

$$
\mathcal{S}[x(\tau)]=\frac{1}{2} \Delta V-\frac{1}{2} f \Delta x+\frac{1}{4} f^{2} t+\int_{0}^{t} \mathrm{~d} \tau \mathcal{L}(\tau) .
$$

The last term

$$
\mathcal{L}(x, \dot{x})=\frac{1}{4} \dot{x}^{2}-U(x)
$$

takes on the form of a Lagrangian for a classical particle with mass $m=1 / 2$ moving in the effective potential

$$
U(x) \equiv \frac{1}{2} V^{\prime \prime}(x)-\frac{1}{4}\left[V^{\prime}(x)\right]^{2}+\frac{1}{2} f V^{\prime}(x) .
$$

It is straightforward to check that for a periodic (and twice differentiable) potential $V(x)$ the effective potential $U(x)$ is periodic, too. For completeness, we note that the same effective potential arises in the symmetrization of the Fokker-Planck operator [Eq. (25)],

$$
e^{V(x) / 2} \hat{L}_{0} e^{-V(x) / 2}=\frac{\partial^{2}}{\partial x^{2}}+U(x),
$$

see, e.g., the textbook treatment in Ref. [28].

In Fig. 3 the effective potential $U(x)$ is plotted for the cosine potential $V(x)=$ $v_{0} \cos (2 \pi x)$ with critical force $f_{\mathrm{c}}=2 \pi v_{0}$. It will turn out useful to define the reduced force $f_{*} \equiv f / f_{\text {c }}$. In the following we will use this specific potential for all illustrations. In equilibrium, the principal feature of the effective potential is that the minimum of $V(x)$ becomes the global maximum of $U(x)$. For $v_{0}>1$, the minimum of $U(x)$ splits into two minima, which are degenerate at equilibrium $(f=0)$. Turning on the driving force $(f>0)$ the global maximum of $U(x)$ is shifted, the depth of the left minimum increases, and the depth of the right minimum decreases until for forces $f_{*} \geqslant\left(1-v_{0}^{-2 / 3}\right)^{3 / 2}$ only one global minimum remains. For $f_{*} \geqslant 1$ the upper arc becomes concave.
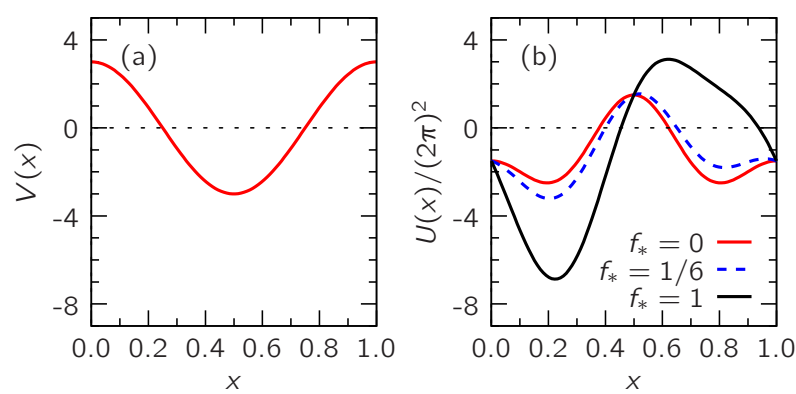

Figure 3. (a) Original potential $V(x)=v_{0} \cos (2 \pi x)$ and (b) the mapped effective potential $U(x)$ from Eq. (34) plotted for $v_{0}=3$ and forces $f=0, f<f_{c}$, and at the critical force $f=f_{\mathrm{c}}=6 \pi$. 


\subsection{Optimal trajectory}

We now determine the trajectory $x_{*}(\tau)$ that minimizes the action [Eq. (32)] with $\mathcal{S}_{*} \equiv \mathcal{S}\left[x_{*}(\tau)\right]$. Clearly, this trajectory is the solution of the Euler-Lagrange equation

$$
-\frac{\delta \mathcal{S}}{\delta x(\tau)}=\frac{\mathrm{d}}{\mathrm{d} \tau} \frac{\partial \mathcal{L}}{\partial \dot{x}}-\frac{\partial \mathcal{L}}{\partial x}=\frac{1}{2} \ddot{x}+U^{\prime}(x)=0
$$

subject to the boundary conditions $x_{*}(0)=x_{0}$ and $x_{*}(t)=x_{0}+\Delta x$. Since the effective potential is periodic, the solution $x_{*}\left(\tau+\tau_{0}\right)=x_{*}(\tau)$ of the Euler-Lagrange Eq. (36) will also be periodic with period

$$
\tau_{0}=\frac{1}{|\sigma|\langle\dot{x}\rangle} .
$$

The sign of $\sigma$ determines the sign of the initial velocity of the particle. Note that the solution of Eq. (36) is time-reversal symmetric.

Before we proceed we shift the effective potential

$$
U(x)=U_{*}+W(x), \quad U_{*}=\max _{x} U(x)
$$

such that $W(x) \leqslant 0$ is non-positive. Although no small parameter appears in the stochastic action, we nevertheless follow the Freidlin-Wentzell route and assume that the transition probability is dominated by the optimal trajectory. We thus obtain from Eq. (32) the unnormalized, naive approximation

$$
\tilde{h}_{*}(\sigma) \equiv \lim _{t \rightarrow \infty} \frac{\mathcal{S}_{*}}{t}=-\frac{1}{2} \sigma\left\langle\dot{s}_{\mathrm{m}}\right\rangle+\frac{1}{4} f^{2}+\frac{A}{\tau_{0}}-U_{*}
$$

with optimal stochastic action for a single period

$$
A \equiv \int_{0}^{\tau_{0}} \mathrm{~d} \tau\left\{\frac{1}{4}\left[\dot{x}_{*}(\tau)\right]^{2}-W\left(x_{*}(\tau)\right)\right\} .
$$

Since the potential $V(x)$ is bounded, the term $\Delta V / t$ drops out in the long time limit. We stress that, since no small parameter appears in the stochastic action, it cannot be expected in general that $h_{*}(\sigma)$ corresponds to $h(\sigma)$ as obtained by the operator method.

The time integral in Eq. (40) is evaluated most conveniently by changing the integration variable from $\tau$ to $x$. To this end we note that

$$
E=\frac{1}{4}\left[\dot{x}_{*}(\tau)\right]^{2}+W\left(x_{*}(\tau)\right)
$$

is a constant of motion. The time to traverse a single period is

$$
\tau_{0}(E)=\frac{1}{2} \int_{0}^{1} \mathrm{~d} x[E-W(x)]^{-1 / 2} .
$$

Rearranging Eq. 40) using Eq. (41), the action for a single period reads

$$
A(E)=\int_{0}^{1} \mathrm{~d} x \frac{E-2 W(x)}{2 \sqrt{E-W(x)}}=\int_{0}^{1} \mathrm{~d} x \sqrt{E-W(x)}-E \tau_{0}(E) .
$$

We thus solve Eq. 42 for a given time $\tau_{0}$ in order to obtain $E$, from which we determine the optimal action $A$. The resulting normalized function

$$
h_{*}(\sigma)=\tilde{h}_{*}(\sigma) /\left\langle\dot{s}_{\mathrm{m}}\right\rangle
$$



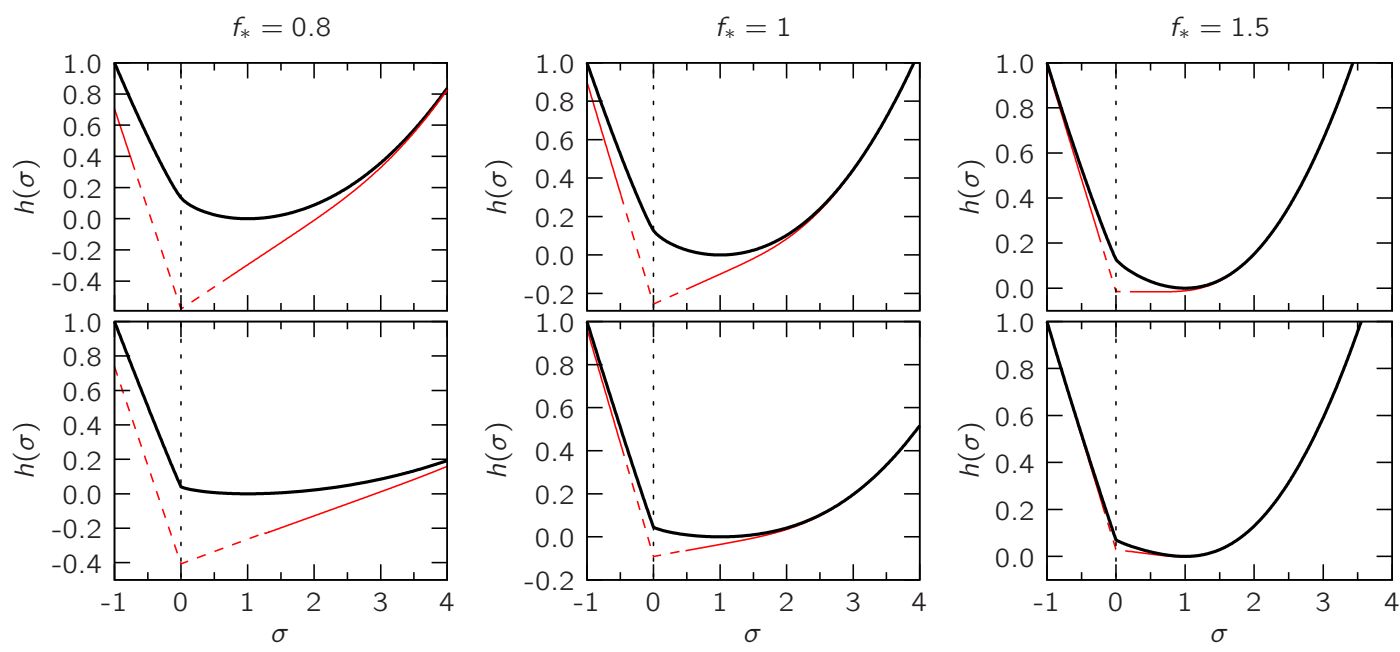

Figure 4. Large deviation function for the periodic potential $V(x)=v_{0} \cos (2 \pi x)$ with $v_{0}=2$ (top row) and $v_{0}=6$ (bottom row) for three reduced driving forces $f_{*}$. Shown are the exact solutions $h(\sigma)$ (thick lines) obtained from the operator method together with the naive approximations $h_{*}(\sigma)$ [Eq. (44), thin lines]. For small $\sigma$ the numerical calculation breaks down and the dashed lines show the linear extrapolation towards the known value $h_{*}(0)$ [see Eq. 49] ].

is plotted in Fig. 4 for different driving forces $f$ below and above the critical force $f_{\mathrm{c}}$. The calculation breaks down for small $\sigma$ as the initial velocity, and therefore $E$, become of the order of the machine precision. We find that for large $\sigma$ the optimal path solution agrees with the full solution $h(\sigma)$ but deviates for small $\sigma$, where the disagreement becomes more pronounced for small driving forces.

We now consider two special cases. For large $E$ the influence of the potential is negligible and the velocity $\dot{x}_{*} \approx \pm 2 \sqrt{E}$ is approximately constant, with $\dot{x}_{*}= \pm 1 / \tau_{0}=$ $\sigma\langle\dot{x}\rangle$. Together with $A=E \tau_{0}$ we obtain from Eq. (39)

$$
\tilde{h}_{*}(\sigma)=\frac{1}{4}(\sigma\langle\dot{x}\rangle-f)^{2}-U_{*},
$$

i.e., the asymptotic form of the large deviation function for large rates $|\sigma|$ is a parabola. In particular, for a flat potential $V(x)=0$ with $\langle\dot{x}\rangle=f$ we recover the exact result $h(\sigma)=(1 / 4)(\sigma-1)^{2}$.

For $E \rightarrow 0$ the period $\tau_{0}$ increases dramatically and the particle spends a long time at the barriers of the effective potential [corresponding to the valleys of the original potential $V(x)$ ], see Fig. 5. Up to linear order in $E$ the optimal action Eq. (43) becomes

$$
A \simeq A_{0} \equiv A(0)=\int_{0}^{1} \mathrm{~d} x \sqrt{-W(x)} .
$$

This is of course the action of an instanton, the classical solution for $E=0$ that connects two adjacent barriers in infinite time. To approximately calculate the period $\tau_{0}$ we expand $W(x)$ to second order,

$$
\frac{\tau_{0}}{2} \approx \frac{1}{2} \int_{0}^{\frac{1}{2}} \mathrm{~d} x\left[E-U_{\mathrm{b}}^{\prime \prime} x^{2} / 2\right]^{-1 / 2},
$$



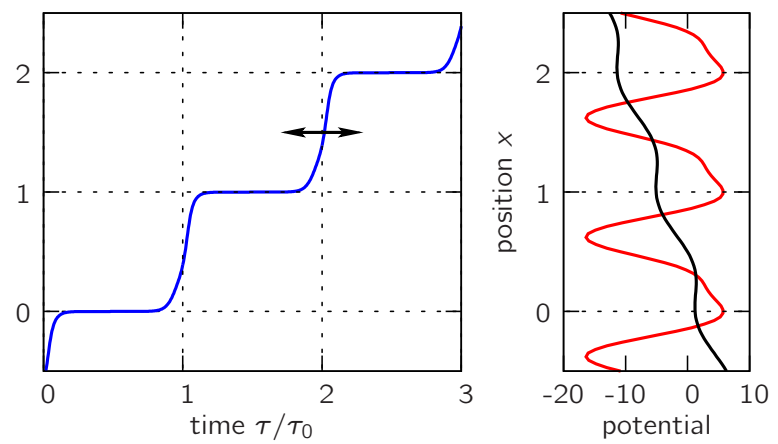

Figure 5. Optimal trajectory $x_{*}(\tau)$ plotted over three periods (parameters: $v_{0}=5$, $f=8 \pi, \sigma=1)$. The potentials $U(x)$ (red/bright) and $V(x)-f x$ (black) are shown in the right panel.

where, without loss of generality, we have shifted the potential along the $x$-axis such that $W(0)=0$, and $U_{\mathrm{b}}^{\prime \prime}<0$ is the curvature of the effective potential at the barrier. To leading order we find the well-known result 31]

$$
E \simeq \frac{\left|U_{\mathrm{b}}^{\prime \prime}\right|}{2} \exp \left\{-\sqrt{2 \mid U_{\mathrm{b}}^{\prime \prime}} \mid \tau_{0}\right\} .
$$

While the time $\tau_{0}$ to traverse a single period diverges for $\sigma \rightarrow 0$, the action of an instanton remains finite and $A_{0} / \tau_{0} \rightarrow 0$. The approximated large deviation function at vanishing entropy production $\sigma=0$ thus reads

$$
\tilde{h}_{*}(0)=\frac{1}{4} f^{2}-U_{*} .
$$

The linear extrapolation of the numerically determined $h_{*}(\sigma)$ towards this value is shown in Fig. 4 .

Summarizing, the results of naively applying the Freidlin-Wentzell approach to the calculation of the large deviation function are: (i) The optimal path is not sufficient to reproduce the large deviation function over the full range of rates $\sigma$. (ii) Only accounting for the contribution from the optimal path results in a function $h_{*}(\sigma)$ that is non-differentiable at $\sigma=0$, i.e., the approximated large deviation function exhibits a genuine kink. (iii) The Gallavotti-Cohen symmetry (3) is fulfilled by both $h_{*}(\sigma)$ and $h(\sigma)$. The rounding of the kink in the full solution $h(\sigma)$ points to the importance of fluctuations around the optimal path even in the limit $t \rightarrow \infty$.

\subsection{Small-noise limit}

As mentioned in the introduction, the Freidlin-Wentzell method is strictly valid only in the limit $\varepsilon \rightarrow 0$ of vanishing noise. In our dimensionless units, the small parameter $\varepsilon=1 / v_{0}$ is the ratio of thermal energy to the potential depth. Fixing $f_{*}$ with $f_{\mathrm{c}} \propto v_{0}$ we see that the first term in Eq. (34) is of order $v_{0}$ and the other two terms are of order $v_{0}^{2}$. We thus drop the first term (the Jacobian). Rearranging terms we obtain for the 

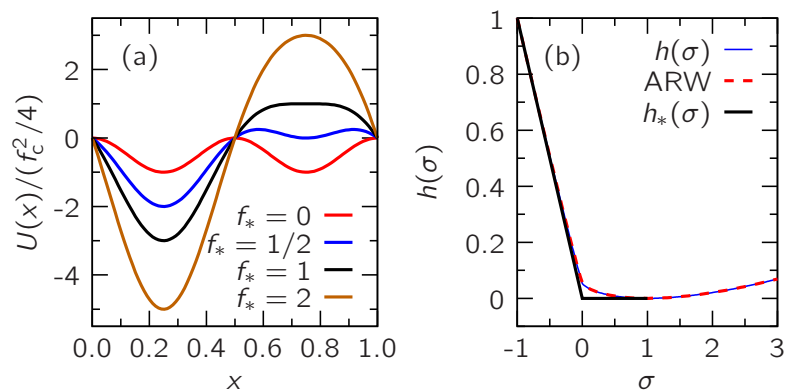

Figure 6. Small-noise limit: (a) Simplified effective potential $U(x)$ dropping the Jacobian in Eq. (34) [cf. Fig. 3(b)]. (b) Approximated LDF $h_{*}(\sigma)$ for $f_{*}<1$ (thick line). Also shown is the LDF $h(\sigma)$ for $v_{0}=7$ and $f=6 \pi\left(f_{*}=3 / 7\right)$ together with the ARW solution Eq. (9p) for the same force.

effective potential the simplified expression

$$
U(x)=\frac{1}{4} f^{2}-\frac{1}{4}\left[-V^{\prime}(x)+f\right]^{2} .
$$

In the following we focus on the case $f<f_{\mathrm{c}}$. The maximum of the potential is then reached at points $x$ where the deterministic force $-V^{\prime}(x)+f$ is zero with

$$
U_{*}=\frac{1}{4} f^{2} \quad\left(f<f_{\mathrm{c}}\right) .
$$

From Eq. 49) we then know that $h_{*}(0)=0$. Since $h_{*}(\sigma)$ is a convex function with $h_{*}(1)=0$ we can infer that the shape in the range $-1 \leqslant \sigma \leqslant 1$ is a "wedge". The approximated LDF is universal in this range and does not depend on $f$ (as long as $\left.f<f_{\mathrm{c}}\right)$ nor $v_{0}$.

Specifically, for the cosine potential $V(x)=v_{0} \cos (2 \pi x)$ the effective potential

$$
U(x)=\frac{f_{\mathrm{c}}^{2}}{4}\left\{f_{*}^{2}-\left[\sin (2 \pi x)+f_{*}\right]^{2}\right\}
$$

is shown in Fig. 6(a), whereas the "wedge"-shaped $h_{*}(\sigma)$ is depicted in Fig. 6(b). The action of an instanton is easily evaluated to give

$$
A_{0}=\int_{0}^{1} \mathrm{~d} x \frac{f_{\mathrm{c}}}{2}\left|\sin (2 \pi x)+f_{*}\right|=\frac{f_{\mathrm{c}}}{\pi}\left[\sqrt{1-f_{*}^{2}}+f_{*} \arcsin f_{*}\right] .
$$

Also shown in Fig. 6(b) is that the actual LDF $h(\sigma)$ agrees with the ARW solution for large $v_{0}$. Since $f_{*}$ is fixed the force $f$ increases as we increase the potential depth $v_{0}$. Both the ARW and the actual solution $h(\sigma)$ then approach the limiting "wedge" shape as $v_{0} \rightarrow \infty$.

\subsection{Role of fluctuations}

At this point it seems natural to inquire what the nature is of the fluctuations contributing to the large deviation function. Specifically, we consider two types of fluctuations: small Gaussian perturbations around the optimal path, and fluctuations of the "jumps" times $\tau_{l}$. In the periodic optimal trajectory $x_{*}(\tau)$ these jumps occur regularly with period $\tau_{0}$, see Fig. 5 . 
3.5.1. Gaussian fluctuations. For finite $t$, we expand the action Eq. (32)

$$
\mathcal{S}\left[x_{*}(\tau)+\xi(\tau)\right] \approx \mathcal{S}_{*}+\frac{1}{2} \int_{0}^{t} \mathrm{~d} \tau \xi(\tau) \hat{D}(\tau) \xi(\tau)
$$

to second order with small perturbations $\xi(\tau)$ of the optimal path, which obey the boundary conditions $\xi(0)=\xi(t)=0$. Here we have introduced the symmetric second variation operator

$$
\hat{D}(\tau) \equiv-\frac{1}{2} \frac{\mathrm{d}^{2}}{\mathrm{~d} \tau^{2}}-U^{\prime \prime}\left(x_{*}(\tau)\right),
$$

which is formally equivalent to a Schrödinger equation with potential energy $-U^{\prime \prime}\left(x_{*}(\tau)\right)$ (positive curvatures of the effective potential correspond to low "energies"). We, therefore, can immediately state that the eigenvalues $\lambda_{i}$ of $\hat{D}$ are real and can be ordered, $\lambda_{0}<\lambda_{1}<\cdots$. Moreover, eigenfunctions $\xi_{i}(\tau)$ corresponding to different eigenvalues $\lambda_{i}$ are orthogonal,

$$
\int_{0}^{t} \mathrm{~d} \tau \xi_{i}(\tau) \xi_{j}(\tau)=\delta_{i j}, \quad \xi_{i}(0)=\xi_{i}(t)=0 .
$$

We expand the generic perturbation $\xi(\tau)$ in the basis spanned by the normalized eigenfunctions $\left\{\xi_{i}\right\}$,

$$
\xi(\tau)=\sum_{i=1}^{\infty} c_{i} \xi_{i}(\tau)
$$

with coefficients $c_{i}$. The path measure is

$$
[\mathrm{d} x(\tau)] \propto \prod_{i=1}^{\infty} \mathrm{d} c_{i}
$$

and we perform the Gaussian integration of Eq. (30) with result

$$
P\left(x_{0}+\Delta x \mid x_{0} ; t\right) \simeq \frac{e^{-\mathcal{S}_{*}}}{\sqrt{\operatorname{det} \hat{D}}}, \quad \operatorname{det} \hat{D}=\prod_{i=1}^{\infty} \lambda_{i} .
$$

Of course, this result only holds if all eigenvalues are positive. We numerically calculate the determinant using Floquet theory as outlined in the appendix. We find that small Gaussian fluctuation around the optimal path $x_{*}(\tau)$ do not contribute to the large deviation function.

3.5.2. Dilute instanton gas. For $f \ll f_{\mathrm{c}}$ the particle in the periodic continuous potential $V(x)$ effectively undergoes a hopping motion resembling the discrete random walk. For such thermally activated jumps, the corresponding rate is

$$
k^{+} \approx \exp \left\{-\Delta V+f\left(x^{+}-x^{-}\right)\right\},
$$

where $x^{-}$is the position of the minimum, $x^{+}$the position of the barrier, and $\Delta V \equiv$ $V\left(x^{+}\right)-V\left(x^{-}\right)$is the barrier height. For the cosine potential we find

$$
2 \pi x^{-}=\pi+\arcsin f_{*}, \quad 2 \pi x^{+}=2 \pi-\arcsin f_{*} .
$$


The rate thus reads

$$
k^{+} \approx e^{-A_{0}+f / 2},
$$

where $A_{0}$ is the action [Eq. (53)] of an instanton in the small noise limit and we have ignored a sub-exponential prefactor.

The optimal trajectory $x_{*}(\tau)$ is strictly periodic. However, Fig. 5 suggests that shifting the positions of the "jumps" $\tau_{l}=l \tau_{0}$ does only minimally change the action as long as the particle spends a quasi-infinite time on top of the barrier (of the effective potential $U$ ). In this case some eigenvalues $\lambda_{i}$ become exponentially small (and therefore contribute to the large deviation function) or even vanish, in which case the calculation of the previous subsection breaks down. Such zero-modes need to be treated in a different way known as the method of collective coordinates. In effect, integrations over zero-modes are replaced by integrations over the corresponding jump time.

Suppose a trajectory is composed of $n^{+}$instantons and $n^{-}$anti-instantons jumping against the driving force. We use again $n=n^{+}-n^{-}$and $m=n^{+}+n^{-}$. If we neglect all "interactions" between instantons we can approximate the action as

$$
\mathcal{S} \approx-\frac{1}{2} f n+m A_{0}
$$

independent of the actual jump times. Integration over the jump times then leads to a path weight

$$
P\left(n^{+}, n^{-} ; t\right)=\frac{1}{Z}\left(\begin{array}{c}
m \\
n^{+}
\end{array}\right) \frac{t^{m}}{m !} e^{-\mathcal{S}}
$$

with normalization factor

$$
Z=\exp \left\{2 t \cosh (f / 2) e^{-A_{0}}\right\} .
$$

We now follow the alternative derivation for the asymmetric random walk presented in Sec. 2.2. Introducing dimensionless fluxes $\nu^{ \pm} \equiv n^{ \pm} /(\kappa t)$ [related by Eq. (17)], we obtain the rate function

$$
\begin{aligned}
J\left(\nu^{+}, \nu^{-}\right)= & 2 \cosh (f / 2) e^{-A_{0}}+\kappa\left\{\nu^{+} \ln \nu^{+}+\nu^{-} \ln \nu^{-}\right. \\
& \left.-(f / 2)\left(\nu^{+}-\nu^{-}\right)-\left(\nu^{+}+\nu^{-}\right)\left(1-A_{0}-\ln \kappa\right)\right\} .
\end{aligned}
$$

The minimum is attained at fluxes

$$
\nu_{*}^{ \pm}=\frac{e^{-A_{0}}}{\kappa}\left[\sqrt{(\zeta \sigma)^{2}+1} \pm \zeta \sigma\right]
$$

with

$$
\zeta \equiv \frac{1}{2} \kappa e^{A_{0}}\left(1-e^{-f}\right)
$$

Plugging in the rate Eq. 62 for $\kappa=k^{+}$we find $\zeta \approx z$ and, moreover, we recover the form Eq. (9) of the LDF for the ARW. 


\section{Conclusions}

We have studied the large deviation function for the entropy production in two simple one-dimensional systems: the asymmetric random walk of a particle on a discrete lattice and the continuous motion of a driven particle in an external potential. For both systems we have calculated the large deviation function using the path approach of Freidlin and Wentzell. However, while for the ARW the solution thus obtained agrees with the Donsker-Varadhan theory, for the continuous case the solution is only an approximation. In both approaches, the wings of the large deviation function are well described by a single trajectory composed of only forward $(\sigma>0)$ or only backward $(\sigma<0)$ jumps. Changing the prescribed rate $\sigma$, the transition between these two regimes appears as a kink at $\sigma=0$, which in the true large deviation function is smeared out by fluctuations. For the ARW the reason is that there are many combinations of forward and backward jumps (and therefore many trajectories) around $\sigma=0$ as described by the combinatorial factor in Eq. (14). For the continuous system we have verified numerically that small perturbations of the optimal path do not contribute to the large deviation function. For large $v_{0}$ and small driving forces $f_{*} \ll 1$ trajectories are dominated by discrete barrier crossings and can be decomposed into instanton solutions. Summing these trajectories, we recover the large deviation function of the ARW.

\section{Acknowledgments}

We thank Hugo Touchette for valuable discussions. TS gratefully acknowledges financial support by the Alexander-von-Humboldt foundation during the initial stage of this work.

\section{Appendix A. Floquet theory}

We estimate the effect of small fluctuations through calculating the full determinant $\operatorname{det} \hat{D}$ of the operator Eq. (55). To this end we make use of the following relation

$$
\frac{\operatorname{det} \hat{D}}{\operatorname{det} \hat{D}_{0}}=\frac{\phi(t)}{\phi_{0}(t)}
$$

with $\hat{D}_{0}$ a suitable reference operator [33. In the simplest case $\hat{D}_{0}=-\partial_{\tau}^{2}$ but the exact form of $\hat{D}_{0}$ does not play a role. Here, $\phi(t)$ is the solution of $\hat{D} \phi=0$ whereas $\phi_{0}(t)$ is the solution of $\hat{D}_{0} \phi_{0}=0$. Defining the vector $\mathbf{x} \equiv(\phi, \dot{\phi})$ we obtain a linear, periodic differential equation

$$
\dot{\mathrm{x}}=\mathrm{M}\left(x_{*}(\tau)\right) \mathbf{x}, \quad \mathrm{M}(x) \equiv\left(\begin{array}{cc}
0 & 1 \\
-2 U^{\prime \prime}(x) & 0
\end{array}\right) .
$$

From Floquet theory it is well-known that the solution can be written

$$
\mathbf{x}(\tau)=c_{+} e^{+\mu \tau} \mathbf{p}(\tau)+c_{-} e^{-\mu \tau} \mathbf{p}(-\tau)
$$


with characteristic, or Floquet, exponent $\mu$. Hence, in the limit of long times the determinant behaves as $\operatorname{det} \hat{D} \sim \phi(t) \sim\left|e^{\mu t}\right|$ to leading order. We again change variables from $\tau$ to $x$ and solve the ordinary differential equation

$$
\frac{\partial \mathbf{X}}{\partial x}=\frac{1}{\dot{x}_{*}} \mathrm{M}(x) \mathrm{X}=\frac{1}{2}[E-U(x)]^{-1 / 2} \mathrm{M}(x) \mathrm{X}
$$

for a matrix $\mathrm{X}$ with the matrix $\mathrm{M}(x)$ given in Eq. A.2). The initial condition is $\mathrm{X}(0)=\mathbf{1}$, and we integrate a single period. The resulting matrix $X(1)$ has two eigenvalues $\rho_{ \pm}=e^{ \pm \mu}$. Numerically we find $\left|\rho_{ \pm}\right| \simeq 1$ for the values of $E$ that can be obtained numerically. Therefore, small Gaussian fluctuations around the optimal path $x_{*}(\tau)$ do not contribute to the large deviation function in the long time limit.

\section{References}

[1] U. Seifert, Rep. Prog. Phys. (in press) arXiv:1205.4176 (2012).

[2] G. Gallavotti and E. G. D. Cohen, Phys. Rev. Lett. 74, 2694 (1995).

[3] J. Kurchan, J. Phys. A: Math. Gen. 31, 3719 (1998).

[4] J. L. Lebowitz and H. Spohn, J. Stat. Phys. 95, 333 (1999).

[5] T. Speck, V. Blickle, C. Bechinger, and U. Seifert, EPL 79, 30002 (2007).

[6] D. Andrieux, P. Gaspard, S. Ciliberto, N. Garnier, S. Joubaud, and A. Petrosyan, Phys. Rev. Lett. 98, 150601 (2007).

[7] F. Douarche, S. Joubaud, N. B. Garnier, A. Petrosyan, and S. Ciliberto, Phys. Rev. Lett. 97, 140603 (2006).

[8] M. Paniconi and Y. Oono, Phys. Rev. E 55, 176 (1997).

[9] A. Saha, J. K. Bhattacharjee, and S. Chakraborty, Phys. Rev. E 83, 011104 (2011).

[10] T. Nemoto and S.-i. Sasa, Phys. Rev. E 84, 061113 (2011).

[11] H. Touchette and R. J. Harris, in Nonequilibrium Statistical Physics of Small Systems: Fluctuation Relations and Beyond, edited by R. Klages, W. Just, and C. Jarzynski (Wiley-VCH, Berlin, 2013), Chap. Large deviation approach to nonequilibrium systems.

[12] T. Speck and U. Seifert, Europhys. Lett. 74, 391 (2006).

[13] V. Blickle, T. Speck, C. Lutz, U. Seifert, and C. Bechinger, Phys. Rev. Lett. 98, 210601 (2007).

[14] U. Seifert and T. Speck, EPL 89, 10007 (2010).

[15] J. Mehl, V. Blickle, U. Seifert, and C. Bechinger, Phys. Rev. E 82, 032401 (2010).

[16] J. R. Gomez-Solano, A. Petrosyan, S. Ciliberto, and C. Maes, J. Stat. Mech.: Theor. Exp. 2011, P01008 (2011).

[17] H. Touchette, Phys. Rep. 478, 1 (2009).

[18] J. Mehl, T. Speck, and U. Seifert, Phys. Rev. E 78, 011123 (2008).

[19] M. Freidlin and A. Wentzell, Random Perturbations of Dynamical Systems, Vol. 260 of Grundlehren der Mathematischen Wissenschaften (Springer-Verlag, New York, 1984).

[20] D. Lacoste, A. W. Lau, and K. Mallick, Phys. Rev. E 78, 011915 (2008).

[21] N. Kumar, S. Ramaswamy, and A. K. Sood, Phys. Rev. Lett. 106, 118001 (2011).

[22] M. Gorissen and C. Vanderzande, J. Phys. A: Math. Theor. 44, 115005 (2011).

[23] V. Lecomte, J. P. Garrahan, and F. van Wijland, J. Phys. A: Math. Theor. 45, 175001 (2012).

[24] T. Speck and J. Garrahan, Eur. Phys. J. B 79, 1 (2011).

[25] S. Dorosz and M. Pleimling, Phys. Rev. E 83, 031107 (2011).

[26] D. ben Avraham, S. Dorosz, and M. Pleimling, Phys. Rev. E 84, 011115 (2011).

[27] A. A. Budini, Phys. Rev. E 84, 061118 (2011).

[28] H. Risken, The Fokker-Planck Equation, 2nd ed. (Springer-Verlag, Berlin, 1989).

[29] D. Lacoste and K. Mallick, Phys. Rev. E 80, 021923 (2009). 
[30] H. Kleinert, Path Integrals in Quantum Mechanics, Statistics, Polymer Physics, and Financial Markets, 5th edition ed. (World Scientific, Singapore, 2009).

[31] B. Caroli, C. Caroli, and B. Roulet, J. Stat. Phys. 26, 83 (1981).

[32] J. Lehmann, P. Reimann, and P. Hänggi, Phys. Rev. E 62, 6282 (2000).

[33] K. Kirsten and A. J. McKane, Ann. Phys. 308, 502 (2003). 